\title{
Isolated Extra hepatic Hydatid Cyst of Para spinal Muscle - Unusual Presentation - a Case Report
}

\author{
Rauniyar R K $\mathbf{K}^{1}$, Sharma $\mathbf{U}^{2}$, Baboo $\mathrm{S}^{3}$ \\ ${ }^{1}$ Department of Radio-diagnosis and Medical imaging and ${ }^{3}$ Department of Orthopaedics, B P \\ Koirala Institute of Health Sciences Dharan, Nepal , ${ }^{2}$ B \&B Hospital, Kathmandu
}

\begin{abstract}
Hydatid disease (echinococcosis) commonly involves liver while other known extrahepatic sites of involvement are lung, heart, brain, kidney, bones, spleen, pancreas, adrenal and muscles. ${ }^{1-3}$ Ultrasonography (US) and computed tomography (CT) features are characteristic especially in hepatic disease and hence diagnosed easily, while isolated extrahepatic diseases are sometimes difficult to diagnose.

Here, we present an interesting case of hydatid cyst in paraspinal muscle with unusual clinical and imaging features, but treated successfully inspite, initial diagnostic dilemma. Imaging features and follow up result are discussed.
\end{abstract}

Keywords: Isolated, Extrahepatic, Hydatid cyst, Paraspinal muscle

\section{Introduction}

Hydatid disease (Echinococcosis) is prevalent throughout the world and is caused by either echinococcus granulosus or alveoloaris. The liver and lungs are the organ most frequently involved. Saidi $\mathrm{F}^{4}$ rule of thumb is $75 \%$ liver, $15 \%$ lung and $10 \%$ rest of body. The liver or lung involvement is most likely if a cyst is present elsewhere in the body ${ }^{4}$. Other extrahepatic sites are lung, heart, brain, kidney, bones, spleen, pancreas, adrenal and muscles. ${ }^{1-3}$

The dog or other carnivore is the definitive host while sheep is the intermediate host.

Correspondence to: Dr. R. K. Rauniyar

Professor and Head,

Department of Radiodiagnosis and Imaging

BPKIHS, Dharan Nepal

Email: rauniyar99@yahoo.com
The adult worm lives in the intestine of the definitive host and discharges eggs which are expelled in the feces. ${ }^{4}$ The intermediate host ingests the ovum while grazing on contaminated ground. The developed embryo rapidly passes through the intestinal wall to enter the portal venous system and hence liver is the organ most frequently involved. The life cycle is completed when an infected intermediate host dies and its viscera which contain the larval form are consumed by a definitive host. Man becomes infected by contact with a definitive host or by consuming contaminated water or vegetables.

The cyst fluid is crystal clear. It is transudate of serum, contains protein and may be antigenic.

As far as diagnosis is concerned serology has little role due to its high false positive or false negative rate up to $15 \%-20 \%$. USG 
and CT scan provide characteristic appearances for diagnosis especially in hepatic disease while extrahepatic disease may pose a diagnostic challenge. Curvilinear calcification on a plain radiograph may be suggestive of disease especially at hepatic and pulmonary site but the diagnosis is best achieved by US and CT which will show daughter cyst and hydatid sand ${ }^{5}$.

We present an interesting case of hydatid cyst in paraspinal muscles with initial diagnostic dilemma but latter treated successfully.

\section{Case History}

A 36 years old female presented with pain mid line lower back of chest for more than a month duration without any history of fever. Clinical examination revealed tenderness over D9. Plain radiograph of dorsolumbar spine AP/Lat. view and chest $\mathrm{x}$-ray were normal. Laboratory examination was unremarkable. Patient was treated symptomatically. No improvement was recorded for a period of month. Patient was taking on and off analgesics for her symptoms. Later CT scan of dorsal spine was done which revealed ill defined hypodense lesion in right paraspinal muscle at D9 level (fig.1). Infective focus/Abscess was suspected and fine needle aspiration cytology (FNAC)/ drainage was advised, but patient refused for any operative intervention. Course of antibiotic and antiinflammatory drugs were given. After 2 weeks she agreed for fine needle aspiration and cytology. Under CT guidance, needle was placed at the lesion (fig.2) and $10-15 \mathrm{ml}$ of water clear fluid was aspirated. Cytological and biochemical examination of fluid was unremarkable. Hydatid cyst was suspected on the basis of characteristic of fluid and patient was given Albendazole $15 \mathrm{mg} / \mathrm{kg}$ once daily for 1 month. Retrospectively US abdomen in view of hepatic involvement was done which did not reveal any abnormality. Pain subsided and latter after 3 months follow up CT scan revealed complete resolution of the lesion.

\section{Discussion}

Hydatid disease (Echinococcosis), primarily affects the liver and the extrahepatic disease is considered to be the consequence of a secondary infection via metastatic spread from the hepatic focus. ${ }^{6}$ Extrahepatic hydatid without hepatic involvement is a rare phenomenon, the explanation given are hepatic omission and passage of oncospheres through hepatic sinuses without carrying disease, passage via lymphatic vessels or via portocaval anastomosis and the vascular passage in a retrograde fashion. ${ }^{6}$ In our patient abdominal US did not reveal any hepatic lesion. As the hydatid disease is mostly located in liver with characteristic appearances allowing a definite diagnosis with US and CT $\operatorname{scan}^{2}$ but the extrahepatic disease may be difficult to diagnose. It is recommended that in extrahepatic cystic tumours hydatid should he considered especially in patients from endemic areas. ${ }^{7}$

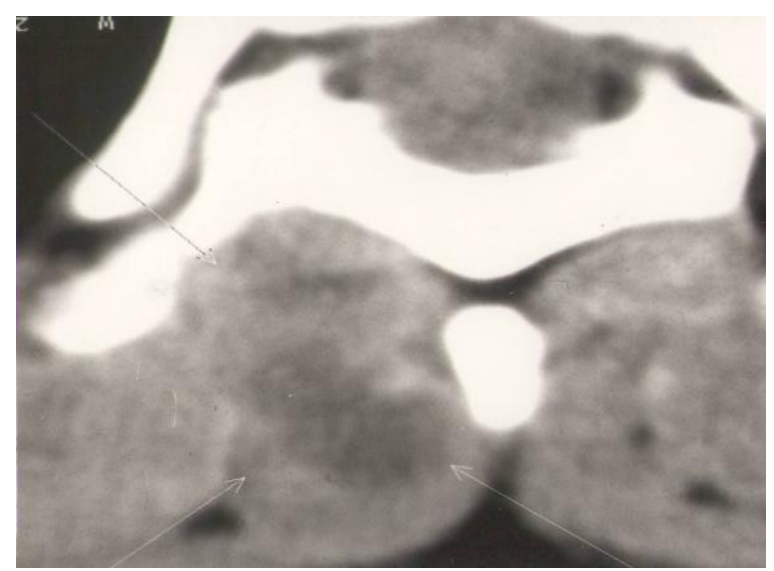

Fig 1: Axial plain CT of dorsal spine in prone position showing ill defined hypodense lesion in right paraspinal muscle.

The common extrahepatic sites reported are lung, heart, brain, kidney, bones, pancreas, adrenal and muscles. ${ }^{1-3}$ Von spinner $\mathrm{WN}$ et al reported a case of primary spinal 
echinococcosus of lumbosacral spine with destruction of the pedicles L3-5 and extension of a large paraspinal cystic mass into the spinal canal. ${ }^{8} \mathrm{CT}$ and US are complementary to each other in diagnosis while serology has little role. It is suggested that the entire abdomen and pelvis should be scanned in patients suspected of hydatid diseases and when a hepatic lesion is suggestive of the disease. ${ }^{9}$ An unusual complication of rupture of hepatic hydatid in to the biliary system with obstructive jaundice has been reported. ${ }^{10}$

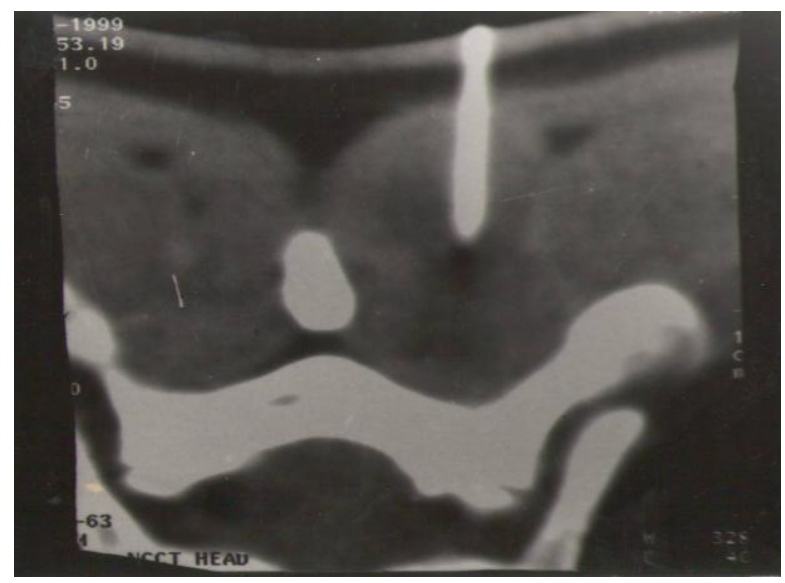

Fig 2: Axial plain CT in prone position showing needle tip in the lesion.

As far as we know isolated hydatid of paraspinal muscle is not reported. The diagnosis of extrahepatic lesion is difficult even with US and CT scan as happened in our case because of lack of characteristic imaging findings. The water clear fluid on aspiration was the only clue for suspicion of the disease and the trial of Albendazole with complete resolution of the lesion confirmed the diagnosis. Our case was interesting and unusual in two ways, firstly this being isolated disease at unusual site in paraspinal muscle (not reported earlier), secondly, due to its unusual USG and CT feature appearing as infective focus/abscess. No clue for hydatid was obtained in our patient from the CT appearances.

\section{Conclusion}

Hepatic and pulmonary hydatid can be diagnosed easily with plain radiography and US, but other extrahepatic disease is a real diagnostic challenge as happened in our case. Our study of this patient highlighted the rare site of involvement of the disease in paraspinal muscle and the diagnostic dilemma even with CT scan. We recommend that on CT scan if clear cyst is not identified FNAC is the next step in diagnosis and if water clear fluid is aspirated hydatid disease should be suspected and trial of Albendazole will confirm the diagnosis.

\section{References}

1. MzabiR, Dziri C, Extrahepatic echinococcosis: diagnosis and treatment. Rew prat; 40(3):220-4,1990.

2. Meyer E, Adam T, Unusual manifestations of echinococcosis. Radiologe;29(5):245-9, 1989.

3. Schoeneich G, Heimback D, Buszello H, Muller SC, Isolated echinococcal cyst of the kidney. Case report and review of the literature. Scand J UrolNephrol; 31(1):95-8, 1997.

4. Saidi F. Surgery of hydatid disease philadelphia: Saunders, 1976.

5. Beggs 1, The radiological appearances of hydatid disease of liver. Cliii radiol ;34:555-564, 1983.

6. Reuter s, Seitz HM, Kern P. Junghanss $\mathrm{T}$, Extrahepatic alveolar echinoccosis without liver involvement: a rare manifestation Infection ;28(3): 18792.,2000.

7. Kluppel D, Seeskd HG. Cystic spaceoccupying lesion with involvement of the pelvis: Unusual site of 
echinococcosis. Case report and surgecal treatment chirurg ;72(5): 610-2,2001.

8. Von Sinner WN, Akhtar M, Primary spinal echinococcosis (Echinococcus granulosus) of lumbosacral spine with destruction of the left pedicles of L3-5 and extension of a large paraspinal cystic mass into the spinal canal. Skeletal Radiol ;23(3):220-3,1994.
9. El-Tahir MI, Omojola MF, Malatani T, al-saigh AH, Ogunbivi OA, Hydatid disease of the liver: evaluation of ultrasound and corn pitted tornography. Br J Radiol ;65(773):390-2,1992.

10. Greulich T, Kohier B, Obstructive jaundice caused by spontaneous rupture of an Echinococcus granulosus cyst into the bile duct system. Z Gastroenterol ;38(4):301-6,2000. 